\title{
Biomechanical analysis of structural deformation in living cells
}

\author{
D. L. Bader · M. M. Knight
}

Received: 22 October 2007/Accepted: 21 July 2008/Published online: 26 August 2008

(C) International Federation for Medical and Biological Engineering 2008

\begin{abstract}
Most tissues are subject to some form of physiological mechanical loading which results in deformation of the cells triggering intracellular mechanotransduction pathways. This response to loading is generally essential for the health of the tissue, although more pronounced deformation may result in cell and tissue damage. In order to determine the biological response of cells to loading it is necessary to understand how cells and intracellular structures deform. This paper reviews the various loading systems that have been adopted for studying cell deformation both in situ within tissue explants and in isolated cell culture systems. In particular it describes loading systems which facilitate visualisation and subsequent quantification of cell deformation. The review also describes the associated microscopy and image analysis techniques. The review focuses on deformation of chondrocytes with additional information on a variety of other cell types including neurons, red blood cells, epithelial cells and skin and muscle cells.
\end{abstract}

Keywords Cell deformation - Mechanotransduction · Mechanics $\cdot$ Confocal $\cdot$ Biomechanics

\section{Cell deformation}

All tissues in mammalian organisms are composed of an assembly of cells, most of which are situated in structures subjected to a degree of mechanical loading. The mechanical

D. L. Bader · M. M. Knight ( $\square)$

School of Engineering and Materials Science,

Queen Mary, University of London, Mile End Road,

London E1 4NS, UK

e-mail: m.m.knight@qmul.ac.uk stimuli are transferred to the cells, typically through any associated extracellular matrix (ECM) resulting in deformation to the living cells. This physiological or pathological loading may alter cellular behaviour in a process termed mechanotransduction. For example, applied forces and associated cell deformation induce a variety of cellular processes including growth, differentiation, adaptation and cellular breakdown. Knowledge of the deformation or strain, experienced by the cells within a tissue, can aid in the understanding of these mechanotransduction processes which are vitally important in many aspects of tissue health, damage and disease. Common examples include mechanosensory mechanisms in bone [65], cartilage maintenance and homoeostasis as affected by both static and dynamic compression $[41,66]$ and the formation of atherosclerotic plaques in vascular tissues $[15,51]$. In addition, in vitro mechanical conditioning strategies for cell-seeded scaffolds, have been proposed as an essential feature for ensuring the long term functionality of tissue engineered implants, such as those for ligament [1] and cartilage [37, 54, 79]. This requires the development of suitable bioreactors, incorporating mechanical loading modules for use in a controlled biological environment. Accordingly, the manner in which tissues and cells respond to different mechanical environments has attracted a wide interest. Many studies have investigated the influence of mechanical loading on different aspects of cell function and the associated mechanotransduction signalling pathways in a diverse range of cell types. However, one significant criticism of many of these studies is that the researchers have little or no idea of the biomechanical stimuli at a cellular level such as the degree of cell deformation. Without this fundamental knowledge it is difficult to fully elucidate the underlying mechanostransduction signalling mechanisms and pathways. This review therefore examines the analysis of cell deformation and the 
techniques that may be employed for a variety of cell types exposed to different biomechanical stimuli. In addition this review examines techniques that may be used to calculate cellular mechanical properties by combining measurements of cell deformation with precise measurements of applied force or pressure.

\section{Mechanical loading systems for the study of cell deformation}

For several decades, there has been interest in visualising and measuring the deformation of living cells. Measurements generally involve deformation of the cell surface, at least in part, by a known force, stress or applied strain. There are numerous techniques to impart some form of deformation on living cells whilst simultaneously enabling direct microscopic visualisation and/or measurement of cell deformation. There have been many studies describing specialist loading rigs and bioreactor systems designed to apply in vitro mechanical stimuli to cells in order to study their metabolic or injury responses (for review see [10]).

Typically, the deformation of most cells in vivo occurs through the gross mechanical loading of the whole tissue. This tissue deformation is transferred to the cells, either directly, via the extracellular matrix, as in muscle, or indirectly as in bone where loading induced fluid flow causes shear deformation at the cell surface. However, for blood cells, physiological deformation occurs during flow through narrow capillaries [4]. Similarly endothelial cells forming the lining of blood vessels experience deformation through a combination of fluid shear and circumferential mechanical stretch of the tissue [15]. In addition, changes in extracellular osmotic pressures may trigger cell deformation in the form of volumetric changes [13, 21, 34]. Clearly, a range of different loading parameters and in vitro cell based model systems are therefore required to investigate physiological deformation in different cell types.

\subsection{Cell deformation in tissue explants}

Various studies have investigated the cell deformation in tissue explants subjected to mechanical loading in the form of tension, compression or shear. Accordingly, a variety of loading rigs have been developed. These systems, such as the one shown schematically in Fig. 1, typically mount upon the stage of an inverted microscope enabling simultaneously visualisation of the resulting deformation to the tissue and cells. By visualising the tissue in both the unstrained and strained state it is possible to calculate the local strain fields by using the cells as displacement markers, in a similar manner to that described with compressed cartilage explants $[27,71]$. The techniques can be used to quantify the nature of the local strain distributions as illustrated in Fig. 2 which clearly shows the presence of shear strains arising from compression of two opposing cartilage surfaces.

Whatever the tissue type and loading modality, it is clear that the heterogeneous nature of most biological tissues frequently results in heterogeneous levels of local deformation, such that the local tissue strains differ greatly from the applied gross strain. This can be illustrated by analysing the local strains within viable fascicles dissected from tendon explants and subjected to tensile strain in a microscope-mounted loading rig similar to that shown in Fig. 1 [72]. Using the nuclei of the tendon cells as displacement markers, the local strains along the collagen fibres were found to be consistently smaller, never exceeding $1.2 \%$ even at a gross applied strain of $8 \%$.

Where gross mechanical loading induces local tissue deformation, this may be transferred to the cells via the extracellular matrix which interfaces with the cell through

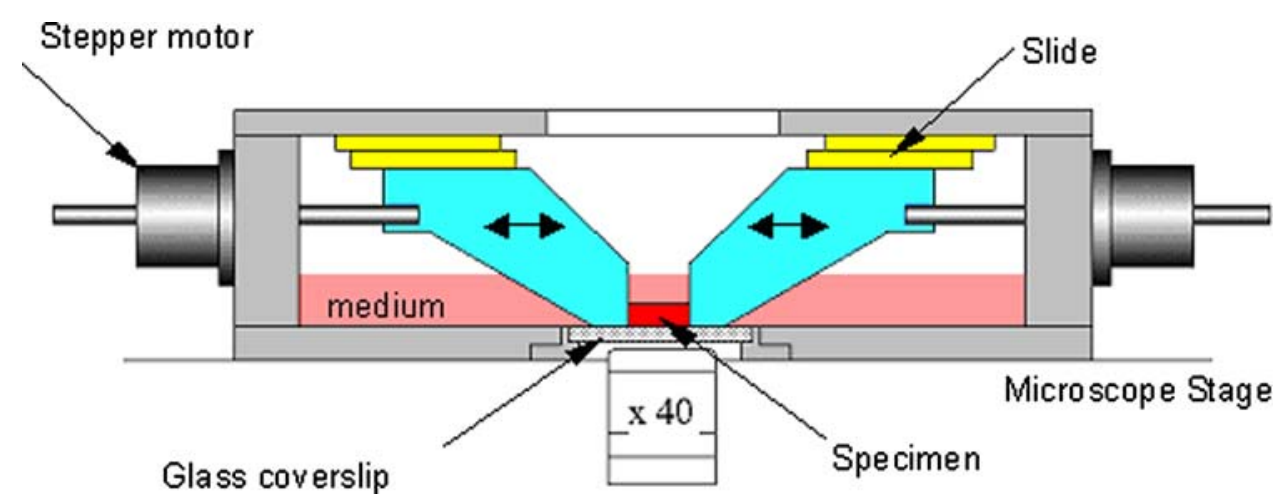

Fig. 1 Schematic cross-sectional view of loading rig for compression of tissue explants or cell seeded scaffold on the stage of an inverted microscope [44]. The specimen is placed on a coverslip and hydrated in medium. Compression is applied via 1 or 2 sliding platens connected to stepping linear actuators controlled via a PC. The original device, developed by Knight, can also be modified to apply tensile strain to tissue explants or cells seeded onto elastomeric membranes [72] 


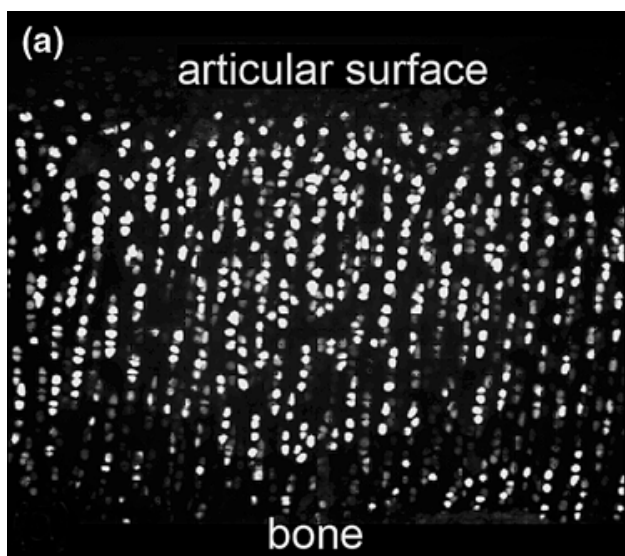

Fig. 2 Confocal images showing an explant of full thickness articular cartilage visualised in the unstrained state (a) and subjected to $20 \%$ static gross compression (b). The explant was taken from the proximal surface of the joint and compressed against an opposing

cell surface receptors and integrins. In the case of intact cartilage explants, gross compressive strain is transferred to the chondrocytes, resulting in cell deformation with an associated reduction in cell volume [25, 27]. This loss of cell volume in situ may result directly from mechanical deformation squeezing fluid from the cell. Alternatively, fluid loss from the compressed extracellular matrix may produce an increase in pericellular osmolarity and hence an osmotic deformation of the cells. Similar cell deformation via mechanical and physicochemical processes may also occur in other tissues.

\subsection{Deformation of isolated cells seeded in 3D scaffolds}

For many cell types, appropriate in vitro model systems involve culturing the living cells within a $3 \mathrm{D}$ scaffold. Accordingly, studies have examined the deformation of the various cell types subjected to mechanical stimuli within 3D scaffold systems. In addition, the increasing interest in tissue engineering techniques has led to the development of cell-seeded scaffolds systems for repair of diseased or damaged tissues including cartilage, bone, ligament, liver and nerve. In order to optimise the design of mechanical conditioning bioreactors used for tissue engineering, it is important to understand the mechanical stimuli perceived by isolated cells in 3D scaffolds. Thus it is necessary to determine the degree of cell deformation and how this varies in space and time. For low modulus scaffolds, such as the widely used hydrogels, agarose and alginate, compressive strain can be applied using relatively simple microscope-mounted loading rigs such as that shown in Fig. 1. These enable simultaneous visualisation of cells at different levels of applied gross compression. Equivalent systems can also be used to apply tensile strain to cell

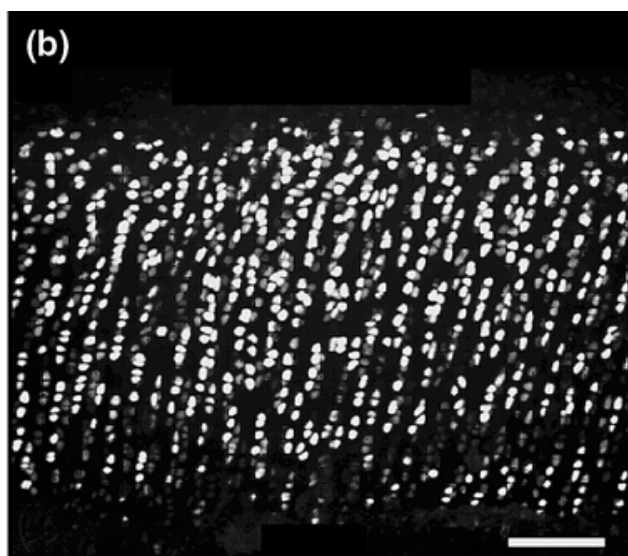

explant from the distal surface. The image shows cells labelled with Calcein AM in tissue adjacent to the area of initial contact (to the left of the field view). Loading clearly produces both compressive and shear strains. Scale bar represents $100 \mu \mathrm{m}$

seeded scaffolds, although, in this case, the ends of the specimen need to be securely gripped.

Examples of studies investigating cell deformation in compressed 3D scaffolds include the numerous studies using the well-characterised chondrocyte-agarose model to investigate the role of cell deformation in chondrocyte mechanotransduction. This in vitro model system consists of isolated articular chondrocytes seeded within agarose gel, typically prepared at a concentration of $2-4 \%(w / v)$ $[48,49]$. Studies using confocal microscopy to measure the deformation of viable chondrocytes in agarose, demonstrate that gross compression results in deformation from a spherical morphology in the unstrained state, to an oblate ellipsoid morphology. Figure 3, shows a series of confocal images taken of a single chondrocyte labelled with the fluorescent dye Calcein-AM (Molecular Probes) and visualised in an agarose construct in the unstrained state and at incremental levels of gross static compression up to $20 \%$. From these images it is possible to quantify the cell deformation or strain in terms of the percentage change in cell diameters, parallel and perpendicular to the applied gross strain [50]. Where it is not possible to measure the same individual cells in both an unstrained and strained state, a deformation index may be used representing the

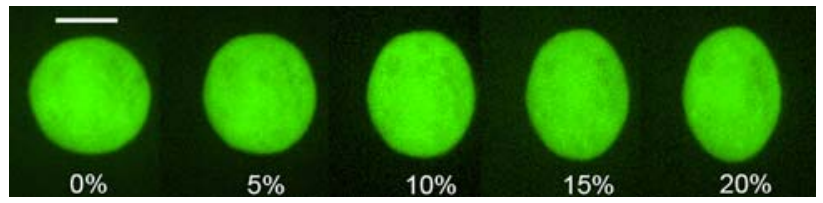

Fig. 3 Confocal images of a single isolated chondrocyte labelled with Calcein AM and visualised in an agarose construct at 0, 5, 10, 15 and $20 \%$ gross compression strain. Images represent a single confocal section bisecting the centre of the cell. Scale bar indicates $5 \mu \mathrm{m}$ 
ratio of the orthogonal cell diameters, with a value of 1 indicating a spherical cell [44].

It is important to appreciate that the nature of cell deformation in 3D scaffolds can differ from that experience in the native tissue. This may be the result of difference in the scaffold mechanical or physicochemical properties, differences in cell attachment or the effects of newly elaborated extracellular matrix. For example, compression of freshly isolated chondrocytes in agarose, as shown in Fig. 1, occurs with significant lateral expansion and conservation of cell volume [50] in contrast to the behaviour in cartilage explants [25, 27]. Furthermore, the matrix synthesised by isolated cells can form a pericellular shell which is stiffer than the surrounding agarose and thus prevents cell deformation during gross compression [44]. Furthermore the viscoelastic properties of the scaffold relative to the cell can lead to temporal changes in cell deformation during either static or cyclic compression [43, 45].

Analysis of cell deformation in 3D scaffolds has also been utilised as a means of quantifying cell mechanical properties. An example of this indirect method involves compressing isolated chondrocytes in $2 \%(\mathrm{w} / \mathrm{v})$ alginate gel (GMB low viscosity, Kelco, UK) using the microscope mounted loading rig shown in Fig. 1. By monitoring the reduction in cell deformation over a $60 \mathrm{~min}$ period of static compression and relating this to the viscoelastic stress relaxation in the gel, measured using a $2.5 \mathrm{~N}$ load cell, it was possible to estimate the cell compressive modulus ( $E=$ stress/strain) at a value of approximately $3 \mathrm{kPa}[2,3$, 45]. However, this approach does not take into account the long term viscoelastic behaviour of the cell as demonstrated in previous studies [45, 77].

\subsection{Deformation of cells using substrate tension}

It is possible to culture a wide variety of cell types as an adherent monolayer without disruption of the cellular phenotype. For example fibroblasts, tenocytes, osteoblasts, hepatocytes, neurons and stem cells may all be cultured in monolayer within standard cell culture flasks. For these cell types, tensile mechanical stimuli may be applied by culturing the cells on a deformable substrate. For the application of relatively small strains, typically less than $1 \%$, a 4 point bend configuration has been adopted [70]. However, by far the commonest approach is to culture cells on an elastomeric membrane. The first elastomeric-bottomed circular plate developed specifically for mechanostimulus work in cell culture, was described in 1985 [5]. The design incorporated a vacuum manifold which, when activated, stretched the under surface of a substrate in a downwards direction, thus imparting a biaxial strain to the culture layer. The system was commercialised under the name Flexercell ${ }^{\mathrm{TM}}$ (http://flexcellint.com/), and has been subsequently modified to apply a more homogeneous, and, where appropriate, a uniaxial, tensile strain field. Other noncommercial systems have now been developed based on the same principle of applying strain to cells by deformation of the substrate to which the cells are attached (for review see [10]). However, for all loading systems it is useful to measure the deformation of the cells to understand the potential mechanotransduction response. For this purpose, Flexcell $^{\mathrm{TM}}$ now produce a membrane based loading rig, Stage Flexer ${ }^{\mathrm{R}}$, which mounts on the stage of an upright microscope. Alternatively, the system shown in Fig. 1 may be modified to apply tensile strain to cell seeded elastomeric membranes. Unlike the Stage Flexer ${ }^{\mathrm{R}}$, this loading system is compatible with high numerical aperture (N.A.), oil immersion type objectives and thus can be used to produce high resolution images enabling precise analysis of cellular and intracellular deformation. For example, studies have measured the deformation of isolated peripheral neurons cultured on lamanin coated elastomeric membranes. This loading modality results in deformation to both the cell body and the neurites, as shown in Fig. 4. The cell deformation is applied through the cell focal adhesions triggering physiological and pathological signalling cascades, such as the activation of intracellular calcium signalling which can be simultaneously examined using fluorescent calcium indicators, such as Fluo-4 AM. Images of neurons in the unstrained and strained state may be analysed using computation techniques, such as digital image correlation, which calculate the local deformations throughout the cell as described later. Thus it is possible to explore the correlation between areas of high tensile and bending strains and the localised biological response in order to identify the mechanical dose response characteristics.

\subsection{Deformation of cells subjected to fluid shear forces}

The application of fluid flow and resulting shear stress at the tissue-fluid interface generates a degree of cell deformation. The influence of fluid shear stress has been most widely examined in relation to vascular cells, however fluid shear also occurs during compressive loading of musculoskeletal tissues such as cartilage and bone. Consequently, other studies have examined the influence of fluid shear on these non-vascular cell types. Both cone-and-plate and parallel plate flow chambers have been employed in conjunction with cells cultured in monolayer [15, 16, 69]. Important studies have utilised computational finite element modelling techniques to calculate the cell deformation associated with different magnitudes of fluid shear stress [55]. This biophysical modelling approach utilises existing theoretical models, such as the tensegrity model described by Ingber and others [56, 82]. Computational studies reveal how the local deformation varies over the 
Fig. 4 Confocal images of a DRG neuron labelled with Calcein-AM on an elastomeric membrane at a $0 \%$ and b $5 \%$ tensile strain. c Distortion and deformation of the neurites is highlighted by the overlay image, with green (online only) representing the unstrained state and the overlay red (online only) showing the strained morphology
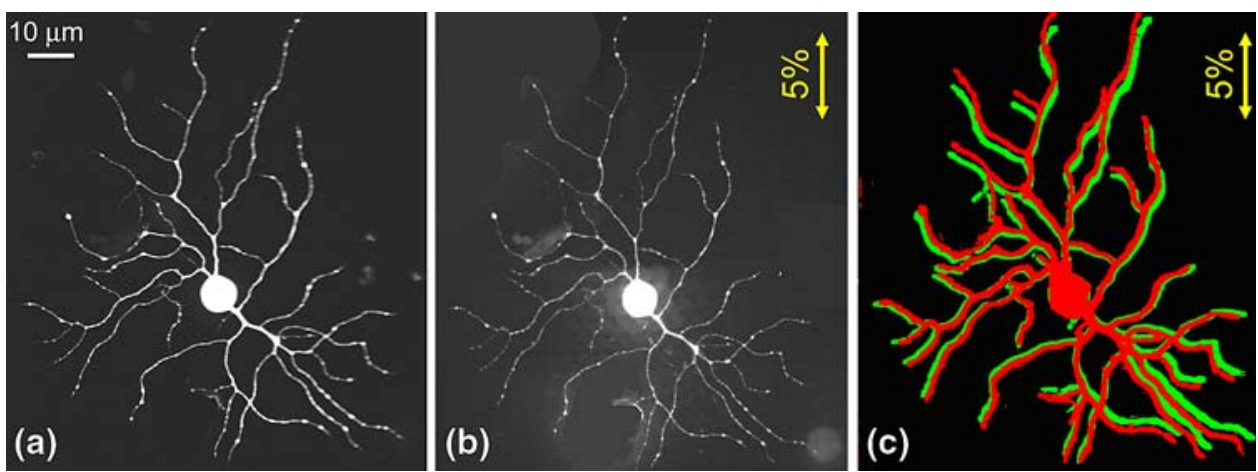

surface of the adherent cell in comparison with that associated with substrate tension as described in Sect. 2.3 (Fig. 5) [55]. In contrast to substrate tension, where the greatest cell strain occurs along the basal surface, fluid shear generates greatest cell distortion on the apical surface. However, even at very low shear stress there may be distortion of trans-membrane cellular components such as the primary cilium or the glycocalx. In deed, fluid shear induced distortion of the primary cilium of kidney epithelial cells is essential for renal function, with disruption of this mechanotransduction process associated with polycystic kidney disease [73].

\subsection{Cell deformation using atomic force microscopy}

Atomic force microscopy (AFM) systems, which were developed from a simple cell poking approach, are now available in both laboratory-based and commercial systems (e.g. Veeco Instruments). They have been employed in a range of applications, primarily in the broad area of nanotechnology and micromechanics. Their use in cell deformation, involves the indentation of the cell surface with a small probe, whose movement is controlled at constant velocity $[64,68]$. The tip, with a radius of curvature of less than $20 \mathrm{~nm}$, is typically pyramidial in shape, $0.6 \mu \mathrm{m}$ in height and $4 \mu \mathrm{m}$ in diagonal length of base. The tip is carefully moved towards the surface of an individual

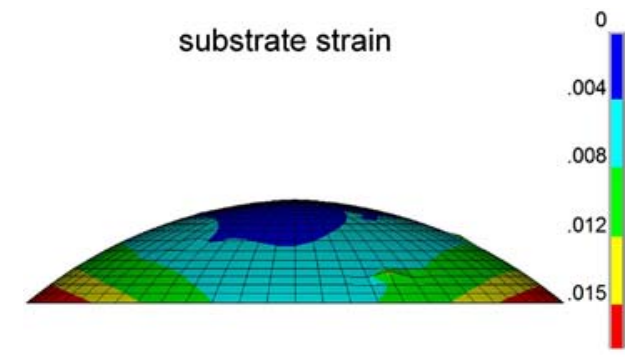

Fig. 5 Finite element model showing the displacement of a single adherent cell subjected to either substrate tension ( $0.1 \%$ strain) or fluid shear $(0.6 \mathrm{~Pa})$. The colour bar represents local displacement cells, imaged through a conventional light microscope. It can interact with various locations on the cell surface with the force indirectly recorded under indentation control of the tip. Hence structural properties, in the form of the force-deformation relationship, can be obtained. However, it should be recognised that interpretation of the results from AFM deformation is complicated by the tapered shape of its probe tip and its small size relative to the depth of indentation. Therefore to determine material properties, such as the cell modulus from this experimental approach, finite element models have been proposed [14]. However, this mode of deformation has little physiological relevance to most cell types.

\subsection{Cell deformation using micropipette aspiration}

An alternative approach employs a micropipette aspiration technique to deform the membrane of the cell with a known suction pressure. Such a technique has been widely used by a number of research groups (for review see [35, 67]). The micropipettes, with inner diameters typically between 5 and $15 \mu \mathrm{m}$, are coated with a silicone solution (Sigmacote, Sigma, MO, USA) to prevent cell adhesion. A cell chamber containing approximately $1 \mathrm{~mL}$ of cell suspension permits the entry of the micropipette through a side wall. The micropipette is fixed to the stage of an inverted microscope and connected to a reservoir and a micro-injector via an

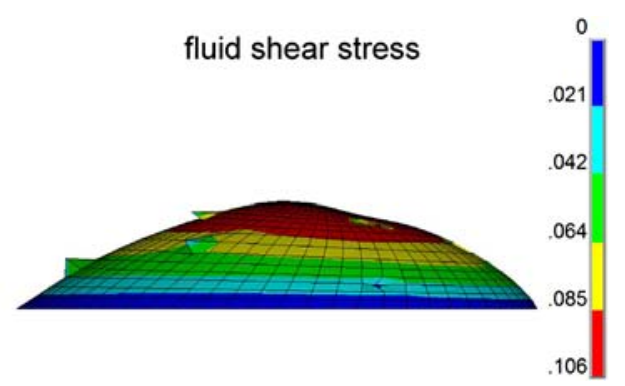

magnitude in $\mu \mathrm{m}$. The image distortion has been exaggerated 30 -fold to illustrate the difference in cellular distortion. Adapted from McGarry et al. [55] 
in-line pressure transducer. Both the micropipette and the pressure control system need to be filled with a physiological saline solution, such as phosphate buffered saline (PBS). With the control of a hydraulic micro-manipulator (e.g. MO-203, Narishige, Tokyo, Japan), the micropipette is moved into contact with the cell surface and pressures applied in a series of step increments, typically up to $5 \mathrm{~cm}$ $\mathrm{H}_{2} \mathrm{O}(0.49 \mathrm{kPa})$ At each pressure increment the cell is allowed to equilibrate for about $60-120 \mathrm{~s}$ before a brightfield microscopy image is taken showing the extent of the cell deformation into the micropipette. This deformation is quantified by the aspirated length, L. A typical series of brightfield images of an isolated cartilage cell subjected to incremental levels of aspiration pressure is shown in Fig. 6 alongside the corresponding confocal fluorescence images showing deformation of the actin cytoskeleton labelled using eGFP transfection. Prior to aspiration, the initial diameter of the cell is measured, as well as the pipette inner diameter using calibrated brightfield microscopy. On the basis of the experimental data, the apparent Young's modulus can be determined using a theoretical model previously developed by Theret et al. to analyse the material properties of endothelial cells [76]. In this model, the cell is assumed to be a homogeneous, elastic half-space material and the Young's modulus, $E$, is therefore given as:

$E=\frac{3 a \Delta P \Phi(\eta)}{2 \pi L}$

where $\mathrm{a}$ and $\mathrm{b}$ are the inner and outer radii of the micropipette and $\Phi(\eta)$ is defined as the wall function with $\eta=(b-a) / a$. In the boundary conditions of this model, the normal displacement of the cell surface at the micropipette-cell contact region is equal to zero, corresponding to $\Phi(\eta)=2.1$ for the practical range used in a typical study. The Young's modulus can be determined from the slope of the linear regression of the normalised length $L / a$ versus the negative pressure $P$. It should be recognised that although this model benefits from being simple and from conditions in which there is a small ratio of micropipette to cell radius, it neglects geometrical factors, such as finite cell dimensions, evolution of cell-micropipette contact region and curvature of the micropipette edges. Thus other models incorporating these geometric factors into a computational form have been developed [29], which will also account for the inhomogeneities in the cellular properties. In addition, other models have been developed specifically for different cell types with more complex morphology such as cochlear outer hair cells [74] and red blood cells [18].

\subsection{Specialised systems for deformation of single isolated cells}

There exist several different techniques for stimulating individual isolated cells through the direct application of mechanical deformation, frequently via a micropipette. However, these so called cell "poking" or "puffing" techniques are non-quantitative, of low repeatability and can not be used to elucidate the influence of different biomechanical loading parameters, such as strain magnitude, rate, frequency and duration. Accordingly, other single cell loading systems have been proposed for examining the role of cytoskeletal actin and the nucleus on the time-dependent compressive behaviour of single muscle cells up to ultimate bursting [60, 61]. In addition, a tensile loading system has also been described, incorporating two micropipettes to grip either side a single fibroblast [59]. One micropipette is fixed to a load cell and the other is attached to a linear actuator providing controlled tensile or compressive strains to the cell. Both tension and compression yield force-deformation curves, which thereby enable measurement of the mechanical and anisotropic properties of the cell.

\section{Intracellular deformation}

Whilst the measurement of cell deformation is essential for analysing gross cell mechanics and potential mechanotransduction mechanisms, an understanding of intracellular deformation provides additional, important information in both these areas. In particular, it can provide clearer
Fig. 6 Brightfield and corresponding confocal microscopy images of a single isolated chondrocyte visualised during micropipette aspiration at pressures of $0,1,2,3$ and $4 \mathrm{~cm}$ of water. Scale bar indicates $5 \mu \mathrm{m}$. Arrows indicate the aspiration of the cell into the micropipette. The cell was transfected with eGFP actin to examine mechanically induced changes in actin remodelling
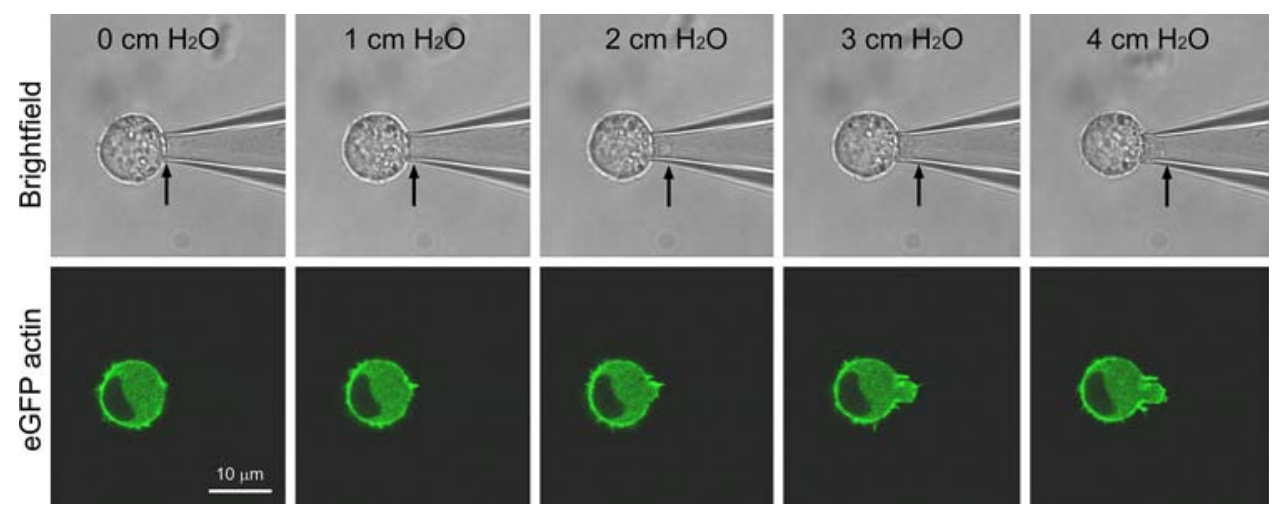
understanding of both the structures which provide cells with their viscoelastic time dependent mechanical properties and the intracellular signalling pathways through which cell deformation is translated into an alteration in cell activity. For example mechanical loading of cartilage induces cell deformation with associated distortion of cellular organelles including the rough endoplasmic reticulum [75], mitochondria [42], nucleus [26] and the primary cilium [40], all of which may have a role in mechanotransduction.

Whilst cell shape may be relatively stable over time, the intracellular environment exhibits significant temporal dynamics. This may occur at both the molecular level, such as the continuous turnover of cytoskeletal proteins, and at the structural level with continuous movement and remodelling. Thus any measurement of intracellular deformation, which occurs over a finite time span, must take into account the inherent temporal dynamics of the system. This is best achieved by limiting the time between unstrained and strained images and comparing measurements of displacement and apparent deformation with those that occurs in unstrained cells over the same time period.

The situation is further complicated by the fact that mechanical stimuli can induce both a direct mechanical deformation on the cell and its intracellular structures as well as an indirect remodelling effect. For example, fluid shear as experience by epithelial cells, creates an initial cell deformation which, in turn, triggers substantial morphological changes, cell reorientation and cytoskeletal remodelling [30]. Care must therefore be taken to distinguish between the direct and indirect effects of mechanical stimuli. This can be partially achieved by considering the time scales over which any cellular or intracellular deformation occurs, with more instantaneous effects most likely to be associated with a direct mechanical deformation. However, this is complicated by the fact that any deformation is likely to be time-dependent due to the viscoelastic properties of the cell $[2,28,39,69,80]$.

Of all the intracellular structures, mechanical deformation of the nucleus has been most commonly examined. Previous studies, using a variety of cell types, indicate that gross compression is transferred from the cell membrane through the cytoplasm to the nucleus [38]. This results in nucleus deformation which may be involved in mechanotransduction through changes in gene expression and nuclear transport [11]. Studies using pipette aspiration to estimate the mechanical properties of the nucleus report that it is approximately 10 times stiffer than the surrounding cytoplasm [28]. Thus, the levels of nucleus deformation are typically less than that of the cell [26, 45, 50]. However, changes in the mechanical properties of the nucleus, for example during differentiation or disease, may result in changes in nucleus deformation and associated alterations in mechanotransduction. In addition, the relative stiffness of the nucleus means that where cell deformation is sufficient to induce nuclear distortion, the nucleus is likely to provide a significant contribution to the gross mechanical stiffness of the cell. Nucleus morphology and deformation are typically heterogeneous and nonuniform, even in situations where the cell morphology and deformation may be considered uniform, such as compression of chondrocytes in 3D constructs [45]. This needs to be considered when quantifying nucleus deformation ideally performed using live cell imaging. Cytoskeletal integrity is important for strain transfer to the nucleus [19, 38] and thus changes in cytoskeletal organisation may also result in alterations in nucleus deformation [45, 50].

\section{Visualisation of cellular and intracellular deformation}

\subsection{Live cell fluorescence microscopy}

The inherent variability in the size, shape and orientation of living cells means that accurate measurement of changes in these parameters is best performed when the same cell can be visualised in both the unstrained and strained state. This therefore precludes the use of techniques such as scanning electron microscopy (SEM) or transmission electron microscopy (TEM) which, although providing high resolution images, require cells to be fixed before imaging. Therefore, in order to quantify the nature and magnitude of cell deformation during physiological mechanical stimuli, studies have adopted various forms of live cell light microscopy. These include techniques such as brightfield, phase contrast or differential interference contrast microscopy as well as epi-fluorescensce and confocal laser scanning microscopy. Of these, the fluorescence-based techniques not only provide clear images of the cell, but are also useful for investigating the deformation of intracellular structures and organelles which can be fluorescently tagged.

The use of fluorescence or confocal microscopy is typically performed using viable fluorescent whole cell stains, such as Calcein AM or Cell Tracker Green (Molecular Probes) [44]. An alternative approach is to saturate the extracellular environment with a low molecular weight fluorescent molecule such as FITC, which is excluded from viable cells. This thereby creates an inverse image of the cell as demonstrated for chondrocytes within intact articular cartilage [27]. Which ever technique is adopted, it is necessary to clearly define the boundary of the cell for precise measurement of cell dimensions and deformation. For fluorescence-based imaging this may be achieved using a high pass digital filter with an intensity threshold set at either a fixed value or a percentage of the maximum intensity [22, 27]. 
For the study of intracellular deformations, the optical sectioning capability of confocal laser scanning microscopy enables clear, blur-free imaging of intracellular structures. The scope of this technology has been greatly enhanced by the every increasing range of fluorescent compounds for labelling intracellular structures and organelles in living cells (see Molecular Probes: http://probes.invitrogen.com/). Thus viable fluorescent markers are now available for organelles including the nucleus, mitochondria and endoplasmic reticulum. Whilst most of these compounds are cell permeable, for labelling of other structures, such as the cytoskeletal protein networks, it is possible to use microinjection of fluorescent analogues (for review see [24]). Alternatively the development of transfection techniques involving fluorescent tags, such as green fluorescent protein (GFP), provides a powerful tool for visualising intracellular structural dynamics and deformation within living cells (Fig. 6).

\subsection{Image resolution}

The resolution of the microscopy system needs to be considered when attempting to measure cellular or intracellular deformation. The theoretical optical limit of spatial resolution for light microscopes is defined using the Rayleigh criterion which describes the separation at which two points are distinctly resolvable. In the lateral, $x-y$ plane this is dependent on the numerical aperture of the objective lens (NA) and the excitation wavelength $(\lambda)$ as shown in Eq. (2).

Lateral limit of resolution for light microscopy $=\frac{0.61 \lambda}{\mathrm{NA}}$.

Thus for a typical x63/1.4NA objective lens and an excitation of $488 \mathrm{~nm}$, the limit of axial resolution is approximately $0.21 \mu \mathrm{m}$. Clearly this is sufficient to measure changes in the shape or size of cells on the micrometer scale (Fig. 3), but insufficient to directly visualise sub-micron level deformation such as localised distortion of the cell membrane. However, the development of fluorescence resonance energy transfer (FRET) techniques or specialised probes such as Laurdan [86] may provide exciting new approaches for measuring deformation and strain beyond the limit of optical resolution.

\subsection{Confocal microscopy for quantification of 3D cell deformation}

It is well known that one of the advantages of confocal microscopy is its ability to produce a series of images at different depths through a specimen, which can be reconstructed to yield a $3 \mathrm{D}$ image. This provides a potential for analysing cell deformation in three dimensions with quantification of parameters, such as cell volume and surface area. However, it is important to note that the axial $z$ resolution is considerably worse than that in the $x-y$ plane as indicated by Eq. (3). Thus the approximate limit of axial resolution for a $\mathrm{x} 63 / 1.4 \mathrm{NA}$ oil immersion objective lens with an excitation of $488 \mathrm{~nm}$ is approximately $0.52 \mu \mathrm{m}$ compared to the lateral resolution of $0.21 \mu \mathrm{m}$.

Axial limit of resolution for confocal microscopy

$$
=\frac{1.4 n \lambda}{(\mathrm{NA})^{2}} \text {. }
$$

Furthermore, axial distortion arises due to any mismatch in the refractive index of the specimen mount medium and the objective immersion medium (e.g. air, water or oil). This necessitates a systematic calibration using fluorescent polymer microspheres of a known diameter [27]. However, mechanical loading is likely to alter the refractive index of a specimen and hence the degree of distortion. In addition, spherical aberration of the objective lens results in an increase in axial distortion with increasing depth into the specimen [53]. Thus although 3D quantitative confocal imaging is certainly possible, it requires a robust systematic calibration to overcome potential artefacts associated with axial distortion and poor lateral resolution. Of course 3D imaging is not essential if the cell can be considered symmetrical in the $z$ plane. Hence for spherical cells, such as isolated chondrocytes subjected to hyper- or hypoosmotic cell deformation, the cell volume can be estimated from measurements of cell diameter $(D)$ or area $(A)$ made from brightfield or 2D confocal images bisecting the centre of the cell [34] (Eq. (4)).

Volume of a spherical cell $=\frac{\pi D^{3}}{6}=\frac{4}{3} \sqrt{\pi A^{3}}$.

An interesting alternative approach for quantifying cell volume changes can be performed by measuring the average fluorescent intensity within cells labelled with the fluorescent stain 5-chloromethylfluorescein diacetate (CMFDA, Molecular Probes) [22]. Changes in cell volume associated with the transport of water across the cell membrane, produce changes in the concentration of the fluorescent stain and hence the intensity of the fluorescent images.

\subsection{Digital image correlation for analysis of intracellular deformation}

The complexity and heterogeneity of intracellular structures, such as the cytoskeleton, requires advanced computational techniques, such as Digital Image Correlation (DIC), to quantify the local deformation. DIC can analyse, with sub-pixel resolution, the movement and 
distortion within pairs of images and has been used for measuring deformation in a wide range of structures including polymeric foams [83], articular cartilage [12, 81] and trabecular bone [57]. More recently, the technique has been optimised for measuring the deformation of fluorescently labelled intracellular structures, visualised in living cells using confocal microscopy [17, 31-33, 36]. Intracellular displacements and strains can be automatically computed and graphically displayed in the form of pseudocolour maps (Fig. 7). The technique is able to determine the local compressive and tensile strains as well as shear strains, area strains, von-Mises strains and the magnitude and direction of the principle strains. However, the technique can not distinguish between percentage changes in dimensions resulting from true mechanical deformation, or strain, and inherent temporal movement or remodelling. However, with appropriate experimental controls it is possible to use this information to generate computational models to describe cellular mechanical properties [6].

\section{Implications of cell deformation in health and disease}

\subsection{Nerve tissue}

Neurons in the peripheral nervous system (PNS) have axons that lie mainly outside the vertebral column and hence

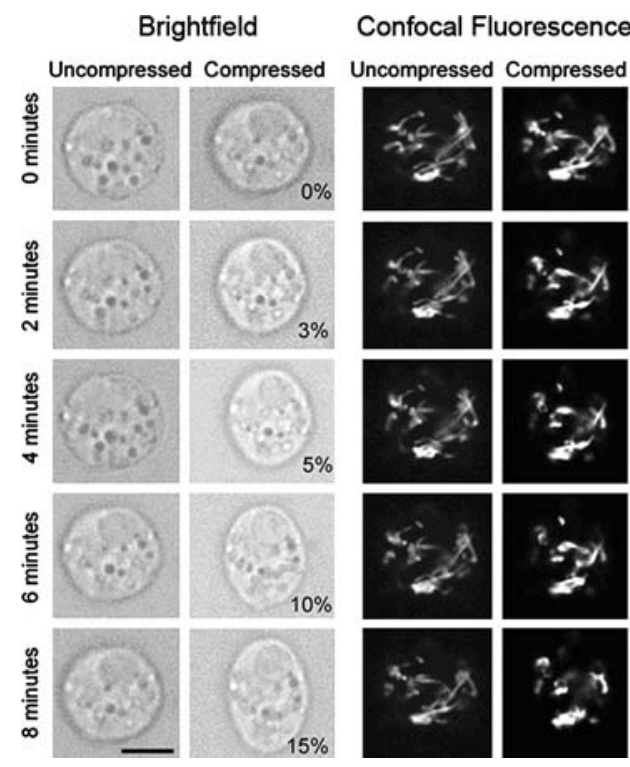

Fig. 7 Brightfield and confocal fluorescence images bisecting the centre of an individual chondrocyte with fluorescently labelled mitochondria, in an agarose construct. The cell was first visualised in an uncompressed state, with pairs of brightfield and confocal images made at $2 \mathrm{~min}$ intervals over an $8 \mathrm{~min}$ period. The construct was then subjected to increments of gross compressive strain, applied every $2 \mathrm{~min}$, up to $15 \%$ strain. Further pairs of brightfield and are subject to a continual dynamic mechanical environment [52]. The axonal endings of these neurons lie in the skin, muscle and viscera and are subjected to a complex array of compressive, tensile and shear strains. Indeed, some PNS neurons act as specialised mechanoreceptors whose function is to detect these mechanical stimuli through a specialised mechanotransduction pathway involving localised cell deformation. In addition, the axonal shafts of peripheral neurons may experience considerable tensile deformation during physiological joint articulation. For example, gross tensile strains of up to $18 \%$ have been measured in human median nerve bed [58], although it is unclear the extent to which this is accommodated by localised sliding of the nerve. Undoubtedly however, the tensile cell deformation of neurons during joint movement is exacerbated by the formation of fibrous adhesions in the nerve root. These can arise due to brachial plexus injury, carpal tunnel syndrome, traumatic crush injuries or following surgery.

Cell deformation can have profound effects on neurons which, depending on the neuronal type and the nature of the loading, can range from induction of neuronal signalling [62], promotion of neurite outgrowth [47] or cell death [85]. While considerable progress has been made in elucidating cellular mechanisms of transduction for light, sound, odour and taste, the role of cell deformation in neuronal mechanotransduction and the associated signalling pathways are as yet unclear.

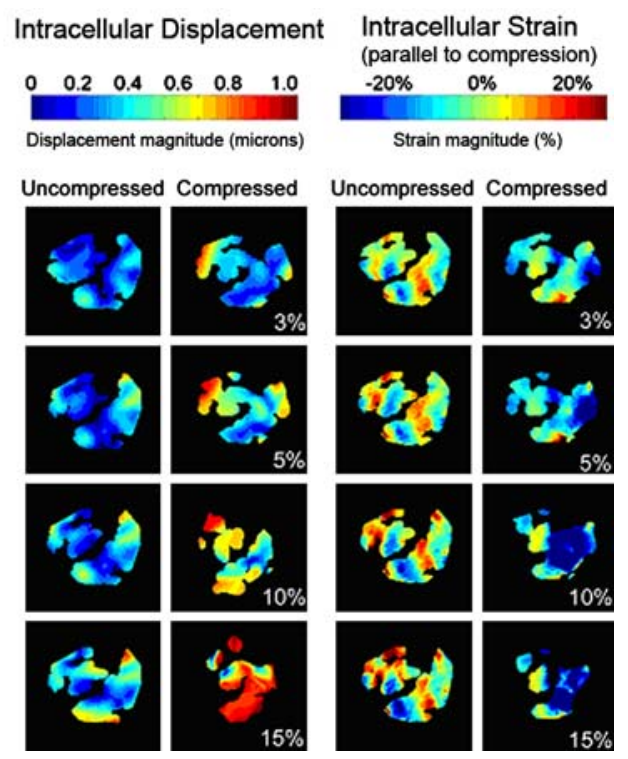

confocal images were recorded at each strain increment. The associated intracellular displacements and strains were calculated, relative to the uncompressed images at $t=0 \mathrm{~min}$, using digital image correlation. The magnitudes of these displacements and strains are shown on a pseudocolour scale. Scale bar indicates $5 \mu \mathrm{m}$. Adapted from [42] 


\subsection{Muscle}

Muscle tissue is particularly susceptible to prolonged tissue compression leading to the development of pressure ulcers. It is well established that skeletal muscle degeneration starts at the cellular level and is characterised by nuclear pyknosis and an early disintegration of the contractile proteins in the cells, followed by inflammatory reactions and tissue necrosis. Although it is clear that both the magnitude and duration of compression affect cellular breakdown, the underlying mechanisms whereby tissue compression results in cell damage are poorly understood. Theories on impaired perfusion and transport of nutrients and metabolic waste products across the cell membrane can only partly explain the onset of tissue damage and have, to date, not been fully verified [20, 46, 78]. It has been hypothesised that sustained deformation of the muscle cells in the tissue plays an additional role in this process. Cell deformation triggers a variety of effects which may be involved in early cell damage, such as local membrane stresses which may lead to buckling and bursting of the membrane, volume changes, and modifications of cytoskeletal organisation affecting its integrity [63]. In addition, changes in the mechanical and chemical environment of the cell may induce further damage. A contribution of cell deformation to mechanotransduction, which influences cell damage and adaptation, might be expected in the presence of compressive strains.

This may be illustrated using an in vitro system consisting of cultured muscle cells embedded in agarose gel, a. system which offers the potential for reproducible, well characterised specimens [7]. The model system has demonstrated that a prolonged compression period will increase the proportion of damaged muscle cells [84]. It is tempting to extrapolate this cell deformation data to the clinical setting, where tissue breakdown, in the form of pressure ulcers, may be induced within relatively short periods of continuous loading. In addition, the model has recently been modified to examine the relative temporal influence of cell deformation and hypoxia on tissue engineered skeletal muscle [23]. It utilised a three-dimensional in-vitro system, incorporating cultured muscle cells seeded in an agarose gel construct. Strain applied to the translucent constructs resulted in deformation of the muscle cells to an elliptical form, which can be quantified using confocal laser scanning microscopy. After a period of compression, sections taken from the central horizontal plane of the individual constructs were stained using both histological and fluorescent probes, to assess the proportion of damage. It was found that constructs subjected to the strain values of $20 \%$ produced a significant proportion of non-viable cells compared to the unstrained constructs. The findings implied a relationship between the duration of applied compression and damage to muscle cells seeded in the gel. The model was extended further by developing a more physiological tissue equivalent muscle [8], by suspending premature muscle cells in a collagen scaffold. The muscle cells fused into a branched network of multinucleated, contractile myofibres by the application of appropriate biochemical and mechanical cues. Results indicated that cell death was evident within $1-2 \mathrm{~h}$ at strain values, equivalent to clinically relevant pressures. A further study examined the relative temporal influence on cell deformation and hypoxia on these tissue engineered equivalents [23]. Compression levels at 20 and 40\%, affected tissue viability with the first $22 \mathrm{~h}$, as assessed by stains for both cell necrosis and cell apoptosis. This is illustrated in Fig. 8. By contrast hypoxia at oxygen concentration below $6 \%$ caused an elevation in glucose utilization, accumulation of lactate, suggesting a transition to anaerobic metabolism, but was not associated with an increasing degree of cell death. The relative importance of cell deformation decreased following prolonged experimentation to 5 days, although in combination with lower $\mathrm{pH}$ values, it continued to result in cell damage.

In addition, the uniform distribution of dead cells throughout the muscle constructs suggested that sustained deformation was the principle cause of cell death. A hybrid approach was then adopted by the authors in which this experimental data was used in the derivation of a damage law [9]. In particular, the evolution of damage was predicted in a single microstructural unit, which could be extrapolated to the macroscopic scale. A damage evolution parameter $D$ was defined, which accumulates with time when the dimensionless strain energy density parameter, $U$, in a cell is higher than a cell tolerance parameter, a. The authors proposed a damage evolution equation (Eq. (5)), where both $\mathrm{a}$ and $\mathrm{b}$ are material parameters which can be determined from the in-vitro experiment:

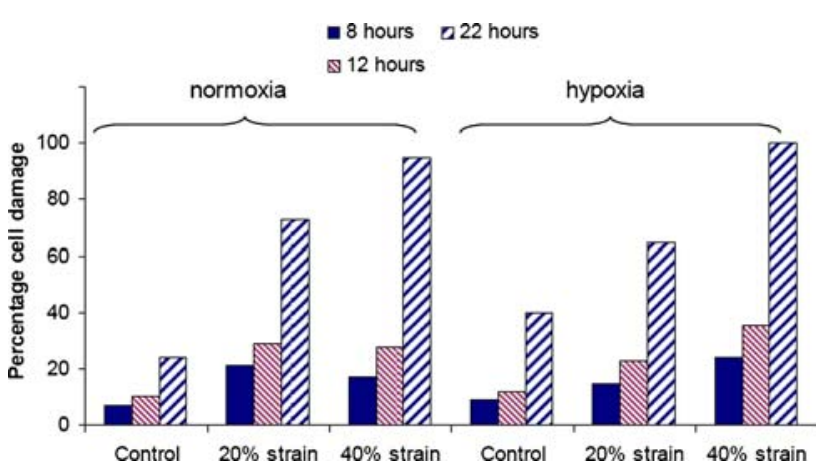

Fig. 8 The percentage of viable muscle cells subjected to compressive strain and/or hypoxia ( $<6 \%$ oxygen tension) at three different culture times. Adapted from [23] 
$D=\int_{0}^{t} \beta(U-\alpha) \mathrm{d} t$.

Although limited at the present time to qualitative insight into tissue damage, this multilevel FE approach has future potential as a quantitative predictor of damage in patientrelated simulations.

\section{Conclusions}

It is evident that physiological mechanical loading is important in the development, health and homeostasis for a wide range of biological tissues. However the associated mechanotransduction pathways, through which the cells detect and respond to their mechanical environment, are as yet, unclear. In addition, the response of cells to mechanical injury is often poorly understood despite the fact that it is critical to the development of strategies to prevent the occurrence of damage or to elicit tissue remodelling. Moreover, the translation of gross mechanical loading of a tissue into cell deformation will determine the ultimate response of the tissue to mechanical loading. Fundamental to these issues is an understanding of how cells deform in response to mechanical loading and the study of cellular biomechanics. Additionally, in order to understand the associated cellular mechanotransduction pathways it is necessary to understand the deformation of specific cellular structures, such as the nucleus and the primary cilium. The emergence of new technology, such as confocal microscopy, GFP transfection, and computational image analysis, provides a powerful toolkit for visualising and quantifying cellular and sub-cellular deformation. Such a multidisciplinary approach can clarify the mysteries of cellular mechanotransduction and mechanical injury and the underlying importance of cell deformation in mechanobiology.

\section{References}

1. Altman GH, Lu HH, Horan RL, Calabro T, Ryder D et al (2002) Advanced bioreactor with controlled application of multidimensional strain for tissue engineering. J Biomech Eng 124:742-749. doi:10.1115/1.1519280

2. Baaijens FP, Trickey WR, Laursen TA, Guilak F (2005) Large deformation finite element analysis of micropipette aspiration to determine the mechanical properties of the chondrocyte. Ann Biomed Eng 33:494-501. doi:10.1007/s10439-005-2506-3

3. Bader DL, Ohashi T, Knight MM, Lee DA, Sato M (2002) Deformation properties of articular chondrocytes: a critique of three separate techniques. Biorheology 39:69-78

4. Bagge U, Branemark PI, Karlsson R, Skalak R (1980) Threedimensional observations of red blood cell deformation in capillaries. Blood Cells 6:231-239
5. Banes AJ, Gilbert J, Taylor D, Monbureau O (1985) A new vacuum-operated stress-providing instrument that applies static or variable duration cyclic tension or compression to cells in vitro. J Cell Sci 75:35-42

6. Bomzon Z, Knight MM, Bader DL, Kimmel E (2006) Mitochondrial dynamics in chondrocytes and their connection to the mechanical properties of the cytoplasm. J Biomech Eng 128:674679. doi:10.1115/1.2246239

7. Bouten CV, Knight MM, Lee DA, Bade DL (2001) Compressive deformation and damage of muscle cell subpopulations in a model system. Ann Biomed Eng 29:153-163. doi:10.1114/1. 1349698

8. Breuls RG, Bouten CV, Oomens CW, Bader DL, Baaijens FP (2003) Compression induced cell damage in engineered muscle tissue: an in vitro model to study pressure ulcer aetiology. Ann Biomed Eng 31:1357-1364. doi:10.1114/1.1624602

9. Breuls RG, Bouten CV, Oomens CW, Bader DL, Baaijens FP (2003) A theoretical analysis of damage evolution in skeletal muscle tissue with reference to pressure ulcer development. J Biomech Eng 125:902-909. doi:10.1115/1.1634287

10. Brown TD (2000) Techniques for mechanical stimulation of cells in vitro: a review. J Biomech 33:3-14. doi:10.1016/S00219290(99)00177-3

11. Buschmann MD, Hunziker EB, Kim YJ, Grodzinsky AJ (1996) Altered aggrecan synthesis correlates with cell and nucleus structure in statically compressed cartilage. J Cell Sci 1996:499-508

12. Chahine NO, Wang CC, Hung CT, Ateshian GA (2004) Anisotropic strain-dependent material properties of bovine articular cartilage in the transitional range from tension to compression. J Biomech 37:1251-1261. doi:10.1016/j.jbiomech.2003.12.008

13. Chao PG, Tang Z, Angelini E, West AC, Costa KD, Hung CT (2005) Dynamic osmotic loading of chondrocytes using a novel microfluidic device. J Biomech 38:1273-1281. doi:10.1016/j. jbiomech.2004.06.016

14. Costa KD, Yin FC (1999) Analysis of indentation: implications for measuring mechanical properties with atomic force microscopy. J Biomech Eng 121:462-471. doi:10.1115/1.2835074

15. Davies PF (1995) Flow-mediated endothelial mechanotransduction. Physiol Rev 75:519-560

16. Deguchi S, Maeda K, Ohashi T, Sato M (2005) Flow-induced hardening of endothelial nucleus as an intracellular stress-bearing organelle. J Biomech 38:1751-1759. doi:10.1016/j.jbiomech. 2005.06.003

17. Delhaas T, Van Engeland S, Broers J, Bouten C, Kuijpers N et al (2002) Quantification of cytoskeletal deformation in living cells based on hierarchical feature vector matching. Am J Physiol Cell Physiol 283:C639-C645

18. Discher DE, Boal DH, Boey SK (1998) Simulations of the erythrocyte cytoskeleton at large deformation. II. Micropipette aspiration. Biophys J 75:1584-1597

19. Djabali K (1999) Cytoskeletal proteins connecting intermediate filaments to cytoplasmic and nuclear periphery. Histol Histopathol 14:501-509

20. Dodd KT, Gross DR (1991) Three-dimensional tissue deformation in subcutaneous tissues overlying bony prominences may help to explain external load transfer to the interstitium. J Biomech 24:11-19. doi:10.1016/0021-9290(91)90322-E

21. Erickson GR, Northrup DL, Guilak F (2003) Hypo-osmotic stress induces calcium-dependent actin reorganization in articular chondrocytes. Osteoarthritis Cartilage 11:187-197. doi:10.1053/ S1063-4584(02)00347-3

22. Errington RJ, Fricker MD, Wood JL, Hall AC, WHite NS (1997) Four-dimensional imaging of living chondrocytes in cartilage using confocal microscopy: a pragmatic approach. Am J Physiol 41:C1040-C1051 
23. Gawlitta D, Oomens CW, Bader DL, Baaijens FP, Bouten CV (2007) Temporal differences in the influence of ischemic factors and deformation on the metabolism of engineered skeletal muscle. J Appl Physiol 103:464-473. doi:10.1152/japplphysiol. 01374.2006

24. Goldman RD, Spector DL (2004) Live cell imaging: a laboratory manual. Cold Spring Harbor Laboratory Press, New York

25. Guilak F (1994) Volume and surface area measurement of viable chondrocytes in situ using geometric modelling of serial confocal sections. J Microsc 173:245-256

26. Guilak F (1995) Compression-induced changes in the shape and volume of the chondrocyte nucleus. J Biomech 28:1529-1541. doi:10.1016/0021-9290(95)00100-X

27. Guilak F, Ratcliffe A, Mow VC (1995) Chondrocyte Deformation and local tissue strain in articular cartilage: a confocal microscopy study. J Orthop Res 13:410-421. doi:10.1002/jor. 1100130315

28. Guilak F, Tedrow JR, Burgkart R (2000) Viscoelastic properties of the cell nucleus. Biochem Biophys Res Commun 269:781786. doi: $10.1006 / \mathrm{bbrc} .2000 .2360$

29. Haider MA, Guilak F (2000) An axisymmetric boundary integral model for incompressible linear viscoelasticity: application to the micropipette aspiration contact problem. J Biomech Eng 122:236-244. doi:10.1115/1.429654

30. Helmke BP, Davies PF (2002) The cytoskeleton under external fluid mechanical forces: hemodynamic forces acting on the endothelium. Ann Biomed Eng 30:284-296. doi:10.1114/1. 1467926

31. Helmke BP, Goldman RD, Davies PF (2000) Rapid displacement of vimentin intermediate filaments in living endothelial cells exposed to flow. Circ Res 86:745-752

32. Helmke BP, Rosen AB, Davies PF (2003) Mapping mechanical strain of an endogenous cytoskeletal network in living endothelial cells. Biophys J 84:2691-2699

33. Helmke BP, Thakker DB, Goldman RD, Davies PF (2001) Spatiotemporal analysis of flow-induced intermediate filament displacement in living endothelial cells. Biophys J 80:184-194

34. Hing WA, Sherwin AF, Poole CA (2002) The influence of the pericellular microenvironment on the chondrocyte response to osmotic challenge. Osteoarthritis Cartilage 10:297-307. doi: 10.1053/joca.2002.0517

35. Hochmuth RM (2000) Micropipette aspiration of living cells. J Biomech 33:15-22. doi:10.1016/S0021-9290(99)00175-X

36. Hu S, Chen J, Fabry B, Numaguchi Y, Gouldstone A et al (2003) Intracellular stress tomography reveals stress focusing and structural anisotropy in cytoskeleton of living cells. Am J Physiol Cell Physiol 285:C1082-C1090

37. Hung CT, Mauck RL, Wang CC, Lima EG, Ateshian GA (2004) A paradigm for functional tissue engineering of articular cartilage via applied physiologic deformational loading. Ann Biomed Eng 32:35-49. doi:10.1023/B:ABME.0000007789.99565.42

38. Janmey PA (1998) The cytoskeleton and cell signalling: Component localization and mechanical coupling. Physiol Rev 78:763-781

39. Janmey PA, Euteneuer U, Traub P, Schliwa M (1991) Viscoelastic properties of vimentin compared with other filamentous bipolymer networks. J Cell Biol 113:155-160. doi:10.1083/jcb. 113.1.155

40. Jensen CG, Poole CA, McGlashan SR, Marko M, Issa ZI et al (2004) Ultrastructural, tomographic and confocal imaging of the chondrocyte primary cilium in situ. Cell Biol Int 28:101-110. doi:10.1016/j.cellbi.2003.11.007

41. Kim YJ, Sah RLY, Grodzinsky AJ, Plaas AHK, Sandy JD (1994) Mechanical regulation of cartilage biosynthetic behaviour: Physical stimuli. Arch Biochem Biophys 311:1-12. doi:10.1006/ abbi.1994.1201
42. Knight MM, Bomzon Z, Kimmel E, Sharma AM, Lee DA, Bader DL (2006) Chondrocyte deformation induces mitochondrial distortion and heterogeneous intracellular strain fields. Biomech Model Mechanobiol 5:180-191. doi:10.1007/s10237-006-0020-7

43. Knight MM, Ghori SA, Lee DA, Bader DL (1998) Measurement of the deformation of isolated chondrocytes in agarose subjected to cyclic compression. Med Eng Phys 20:684-688. doi:10.1016/ S1350-4533(98)00080-0

44. Knight MM, Lee DA, Bader DL (1998) The influence of elaborated pericellular matrix on the deformation of isolated articular chondrocytes cultured in agarose. Biochim Biophys Acta 1405:67-77. doi:10.1016/S0167-4889(98)00102-5

45. Knight MM, van de Breevaart BJ, Lee DA, van Osch GJ, Weinans H, Bader DL (2002) Cell and nucleus deformation in compressed chondrocyte-alginate constructs: temporal changes and calculation of cell modulus. Biochim Biophys Acta 1570:1-8

46. Krouskop TA (1983) A synthesis of the factors that contribute to pressure sore formation. Med Hypotheses 11:255-267. doi: 10.1016/0306-9877(83)90067-1

47. Lamoureux P, Zheng J, Buxbaum RE, Heidemann SR (1992) A cytomechanical investigation of neurite growth on different culture surfaces. J Cell Biol 118:655-661. doi:10.1083/jcb.118.3.655

48. Lee DA, Bader DL (1995) The development and characterisation of an in vitro system to study strain induced cell deformation in isolated chondrocytes. In Vitro Cell Dev Biol 31:828-835. doi: 10.1007/BF02634565

49. Lee DA, Bader DL (1997) Compressive strains at physiological frequencies influence the metabolism of chondrocytes seeded in agarose. J Orthop Res 15:181-188. doi:10.1002/jor.1100150205

50. Lee DA, Knight MM, Bolton JF, Idowu BD, Kayser MV, Bader DL (2000) Chondrocyte deformation within compressed agarose constructs at the cellular and sub-cellular levels. J Biomech 33:81-95. doi:10.1016/S0021-9290(99)00160-8

51. Lee RT, Huang H (2000) Mechanotransduction and arterial smooth muscle cells: new insight into hypertension and atherosclerosis. Ann Med 32:233-235. doi:10.3109/07853890009011765

52. Lundborg G, Dahlin LB (1996) Anatomy, function, and pathophysiology of peripheral nerves and nerve compression. Hand Clin 12:185-193

53. Majlof L, Forsgren P-O (1993) Important considerations for accurate imaging. In: Matsumoto B (ed) Cell biological applications of confocal microscopy. Academic Press, London, vol 38, pp 79-95

54. Mauck RL, Soltz MA, Wang CC, Wong DD, Chao PH et al (2000) Functional tissue engineering of articular cartilage through dynamic loading of chondrocyte-seeded agarose gels. J Biomech Eng 2000:252-260. doi:10.1115/1.429656

55. McGarry JG, Klein-Nulend J, Mullender MG, Prendergast PJ (2005) A comparison of strain and fluid shear stress in stimulating bone cell responses - a computational and experimental study. FASEB J 19:482-484

56. McGarry JG, Prendergast PJ (2004) A three-dimensional finite element model of an adherent eukaryotic cell. Eur Cell Mater 7:27-33

57. McKinley TO, Bay BK (2003) Trabecular bone strain changes associated with subchondral stiffening of the proximal tibia. J Biomech 36:155-163. doi:10.1016/S0021-9290(02)00386-X

58. Millesi $H$ (1986) The nerve gap. Theory and clinical practice. Hand Clin 2:651-663

59. Miyazaki H, Hasegawa Y, Hayashi K (2000) A newly designed tensile tester for cells and its application to fibroblasts. J Biomech 33:97-104. doi:10.1016/S0021-9290(99)00161-X

60. Peeters EA, Bouten CV, Oomens CW, Bader DL, Snoeckx LH, Baaijens FP (2004) Anisotropic, three-dimensional deformation of single attached cells under compression. Ann Biomed Eng 32:1443-1452. doi:10.1114/B:ABME.0000042231.59230.72 
61. Peeters EA, Oomens CW, Bouten CV, Bader DL, Baaijens FP (2005) Viscoelastic properties of single attached cells under compression. J Biomech Eng 127:237-243. doi:10.1115/1. 1865198

62. Perl ER (1992) Function of dorsal root ganglion cells: an overview. In: Scott SA (ed) Sensory neurons. Oxford University Press, New York, pp 3-23

63. Petersen NO, McConnaughey WB, Elson EL (1982) Dependence of locally measured cellular deformability on position on the cell, temperature, and cytochalasin B. Proc Natl Acad Sci USA 79:5327-5331. doi:10.1073/pnas.79.17.5327

64. Radmacher M, Fritz M, Kacher CM, Cleveland JP, Hansma PK (1996) Measuring the viscoelastic properties of human platelets with the atomic force microscope. Biophys J 70:556-567

65. Roelofsen J, Klein-Nulend J, Burger EH (1995) Mechanical stimulation by intermittent hydrostatic compression promotes bone-specific gene expression in vitro. J Biomech 28:1493-1503. doi:10.1016/0021-9290(95)00097-6

66. Sah R, Kim Y, Doong J, Grodzinsky AJ, Plaas AHK, Sandy JD (1989) Biosynthetic response of cartilage explants to dynamic compression. J Orthop Res 7:619-636. doi:10.1002/jor. 1100070502

67. Sato M, Levesque MJ, Nerem RM (1987) An application of the micropipette technique to the measurement of the mechanical properties of cultured bovine aortic endothelial cells. J Biomech Eng 109:27-34

68. Sato M, Nagayama K, Kataoka N, Sasaki M, Hane K (2000) Local mechanical properties measured by atomic force microscopy for cultured bovine endothelial cells exposed to shear stress. J Biomech 33:127-135. doi:10.1016/S0021-9290(99)00178-5

69. Sato M, Ohshima N, Nerem RM (1996) Viscoelastic properties of cultured porcine aortic endothelial cells exposed to shear stress. J Biomech 29:461-467. doi:10.1016/0021-9290(95)00069-0

70. Saunders MM, Taylor AF, Du C, Zhou Z, Pellegrini VD Jr, Donahue HJ (2006) Mechanical stimulation effects on functional end effectors in osteoblastic MG-63 cells. J Biomech 39:14191427. doi:10.1016/j.jbiomech.2005.04.011

71. Schinagl RM, Ting MK, Price JH, Sah RL (1996) Video microscopy to quantitate the inhomogenous strain within articular cartilage during confined compression. Ann Biomed Eng 24:500 512. doi:10.1007/BF02648112

72. Screen HR, Lee DA, Bader DL, Shelton JC (2003) Development of a technique to determine strains in tendons using the cell nuclei. Biorheology 40:361-368

73. Singla V, Reiter JF (2006) The primary cilium as the cell's antenna: signaling at a sensory organelle. Science 313:629-633. doi:10.1126/science. 1124534

74. Spector AA, Brownell WE, Popel AS (1996) A model for cochlear outer hair cell deformations in micropipette aspiration experiments: an analytical solution. Ann Biomed Eng 24:241249. doi:10.1007/BF02667353

75. Szafranski JD, Grodzinsky AJ, Burger E, Gaschen V, Hung HH, Hunziker EB (2004) Chondrocyte mechanotransduction: effects of compression on deformation of intracellular organelles and relevance to cellular biosynthesis. Osteoarthritis Cartilage 12:937-946. doi:10.1016/j.joca.2004.08.004

76. Theret DP, Levesque MJ, Sato M, Nerem RM, Wheeler LT (1988) The application of a homogeneous half-space model in the analysis of endothelial cell micropipette measurements. J Biomech Eng 110:190-199

77. Trickey WR, Vail TP, Guilak F (2004) The role of the cytoskeleton in the viscoelastic properties of human articular chondrocytes. J Orthop Res 22:131-139. doi:10.1016/S07360266(03)0150-5

78. Vohra RK, McCollum CN (1994) Pressure sores. BMJ 309:853857

79. Vunjak-Novakovic G, Obradovic B, Martin I, Bursac PM, Langer R, Freed LE (1998) Dynamic cell seeding of polymer scaffolds for cartilage tissue engineering. Biotechnol Prog 14:193-202. doi:10.1021/bp970120j

80. Wagner O, Zinke J, Dancker P, Grill W, Bereiter-Hahn J (1999) Viscoelastic properties of f-actin, microtubules, f-actin/alphaactinin, and $\mathrm{f}$-actin/hexokinase determined in microliter volumes with a novel nondestructive method. Biophys J 76:2784-2786

81. Wang CC, Chahine NO, Hung CT, Ateshian GA (2003) Optical determination of anisotropic material properties of bovine articular cartilage in compression. J Biomech 36:339-353. doi: 10.1016/S0021-9290(02)00417-7

82. Wang N, Naruse K, Stamenovic D, Fredberg JJ, Mijailovich SM et al (2001) Mechanical behavior in living cells consistent with the tensegrity model. Proc Natl Acad Sci USA 98:7765-7770

83. Wang Y, Cuitino AM (2002) Full-field measurements of heterogeneous deformation patterns on polymeric foams using digital image correlation. Int J Solids Struct 39:3777-3796. doi: 10.1016/S0020-7683(02)00176-2

84. Wang YN, Bouten CV, Lee DA, Bader DL (2005) Compressioninduced damage in a muscle cell model in vitro. Proc Inst Mech Eng $[\mathrm{H}]$ 219:1-12

85. Wolf JA, Stys PK, Lusardi T, Meaney D, Smith DH (2001) Traumatic axonal injury induces calcium influx modulated by tetrodotoxin-sensitive sodium channels. J Neurosci 21:19231930

86. Zhang YL, Frangos JA, Chachisvilis M (2006) Laurdan fluorescence senses mechanical strain in the lipid bilayer membrane. Biochem Biophys Res Commun 347:838-841. doi:10.1016/j. bbrc.2006.06.152 